\title{
Moesin stiffens up mitosis
}

Microtubule spindle dynamics and reorganization of cortical actin drive cell division in animals. Two reports suggest that the ERM (ezrin, radixin and moesin) protein moesin can coordinate these two processes (DOI 10.1016/j.cub.2007.12.051; DOI 10.1083/jcb.200709161).

Moesin, the only known ERM protein in Drosophila melanogaster, crosslinks actin filaments to the plasma membrane and was shown previously to be activated by phosphorylation. Baum and colleagues noticed that phosphorylated moesin accumulated at the cortex of mitotic Drosophila cells in culture, whereas Payre and colleagues observed that moesin-mutant flies exhibited defects in cytokinesis. Both observations suggested a potential role for moesin in regulating cortical actin dynamics during cell division.

Both groups identified Slik as the kinase responsible for moesin activation during mitosis and showed that inactivation of moesin, using RNAi or by silencing Slik, caused mitotic defects. Inactivation of moesin prevented dividing cells from retracting their margins and rounding up, and live analysis revealed that the cortex of moesin-inactivated cells was extremely unstable throughout mitosis. Surprisingly, the mitotic spindles in these cells had variable morphological defects, such as centrosome misplacement and an asymmetrical organization. Erratic changes in spindle positioning accompanied these defects, indicating that moesin also keeps the spindle in place.

Using atomic force microscopy, Baum and colleagues showed that moesin-depleted cells did not undergo the increase in cortical stiffness usually seen in mitosis, and that expression of phosphomimetic moesin in interphase cells was sufficient to induce cortical stiffening and cell rounding. They also demonstrated that incubating moesindepleted cells with concavalin A, which increases cortical stiffness, rescued the defects in spindle morphogenesis and in the cortex during metaphase. Nevertheless, increasing overall cortical stiffness towards

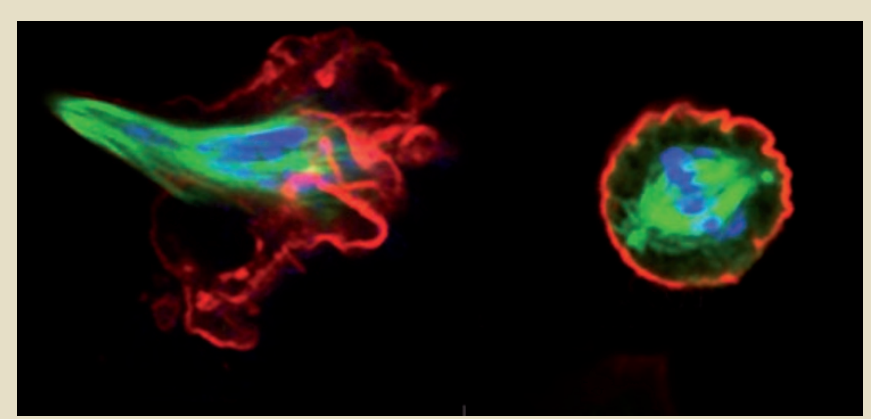

Mitotic spindle and cortical defects in moesin-depleted floating $\mathrm{S} 2 \mathrm{R}+$ cells (left) are rescued by incubation with concavalin A (right), which increases cortical stiffness. Cells were stained for F-actin (red), microtubules (green) and phospho-histone 3 (blue).

the end of mitosis prevented cell elongation, suggesting that moesin activity needs to be regulated precisely in time and space for completion of mitosis.

Both groups showed that the dynamic distribution of F-actin and the non-muscle myosin II motor was impaired when moesin was depleted. Payre and colleagues found that inhibition of RhoA-mediated actin contractility suppressed erratic spindle movements, suggesting that the role of moesin during mitosis, in part, involves RhoA activity. In addition, Baum and colleagues observed that expression of phosphomimetic moesin in cells depleted of myosin II light chain (a known target of RhoA) could induce cortical tension, indicating a role for moesin in cortical stiffening that is independent of myosin II.

Understanding how moesin and myosin II coordinate their efforts to regulate cortical tension, as well as how this, in turn, regulates normal mitotic spindle dynamics will be the challenge of future studies.

Nathalie Le Bot 